\title{
BRIEF
}

\section{Effects of Remote Proctoring on Composite Exam Performance Among Student Pharmacists}

\author{
Elizabeth A. Hall, PharmD, Christina Spivey, PhD, Hailey Kendrex, Dawn E. Havrda, PharmD \\ University of Tennessee Health Science Center College of Pharmacy, Memphis, Tennessee
}

Corresponding Author: Elizabeth A. Hall, PharmD; University of Tennessee Health Science Center College of Pharmacy, 881 Madison Avenue, Memphis, TN 38163. Tel: 901-448-3288. Email: liz.hall@uthsc.edu

Submitted September 30, 2020; accepted January 29, 2021; ePublished February 2021

Objective. To determine the impact of remote proctoring on student pharmacist academic performance.

Methods. This was a retrospective, observational study that compared first- (P1) and second-year (P2) student pharmacist scores on eight composite exams administered in spring $2020(n=387)$, the final three of which were proctored remotely, to that of a historical cohort who took the same exams in spring $2019(\mathrm{n}=368)$. To assess whether remote proctoring affected academic performance, spring 2020 scores for exams 6,7 , and 8 were compared to that of a historical cohort proctored live in spring 2019. Academic performance on exams 1 through 4 was also compared between the two cohorts to evaluate any possible year-to-year variation in academic performance during non-remote circumstances. Mann Whitney tests were used to compare scores between the two cohorts.

Results. Median scores were significantly lower in spring 2020 as compared to the historical cohort on the first composite exam administered to $\mathrm{P} 1$ students after the implementation of remote proctoring. In contrast, median scores were significantly higher for two of three exams administered to P2 students with remote proctoring.

Conclusion. Remote proctoring has a minimal impact on student pharmacist exam performance and may be considered to assure academic honesty and security of testing content in a distance learning environment.

Keywords: academic performance, distance education, proctoring, assessment

\section{INTRODUCTION}

In response to the coronavirus disease 2019 (COVID-19) global pandemic, many educational institutions rapidly transitioned to distance learning. This learning environment presents several challenges, particularly with student assessment and online exams. These challenges may include technical issues, adapting to a novel learning environment, and ensuring academic integrity.$^{1-3}$ One method to assure academic integrity for an exam taken remotely is to institute surveillance measures, such as remote proctoring. ${ }^{4,5}$ Real-time online proctoring services, such as ProctorU (ProctorU, Inc., Birmingham, AL), have recently gained popularity. Milone and colleagues in their study of student pharmacists' perceptions and the impact of ProctorU reported that $88.95 \%$ of student pharmacists were satisfied with their experience. The most frequently encountered issues were technical difficulties, personnel issues, and taking too long to start the exam. ${ }^{5}$ The service also has a fee per exam, which may further limit its utility.

On March 16, 2020, the University of Tennessee Health Science Center (UTHSC) transitioned to a remote learning environment due to the COVID-19 global pandemic. Demand for online proctoring services was at an all-time high, and proctor availability was limited. Consequently, the UTHSC College of Pharmacy developed and executed a novel plan to remotely proctor multiple-choice question exams using only internal and existing resources in April 2020. As remote proctoring was implemented, students anecdotally reported increased levels of stress that they felt were negatively affecting their exam scores.

There are no publications to date investigating how remote proctoring may affect student pharmacists' academic performance. Similarly, the impact of remote proctoring on student performance has not been well described in other health professions. Weiner and colleagues investigated live versus remote proctoring for three high stakes professional licensing exams and found no significant difference in overall scores between the two cohorts. ${ }^{6}$ Given the paucity of existing data, the current study aimed to determine the impact of remote proctoring on academic performance among first(P1) and second-year (P2) student pharmacists.

\section{METHODS}

The remote proctoring process developed and implemented at UTHSC College of Pharmacy used internal resources and existing technology. Remote proctoring required students to use two devices. On the first device, the 
student was monitored with videoconferencing software (Zoom Video Communications, Inc., San Jose, CA). On the second device, the computer-based exam was administered securely via ExamSoft (ExamSoft Worldwide, Inc., Dallas, TX). Secure exams in ExamSoft restrict access to all other applications and disable all device functions during the exam.

Proctors were UTHSC College of Pharmacy faculty and staff who completed a one-hour training that discussed procedures before the exam, during the exam, and after the exam along with a written procedures guide. Additional oneon-one sessions with IT staff were available if needed for adequate orientation. Students also attended a one-hour informational session to learn what to expect on exam day and could consult with IT staff as needed before the exam.

Prior to the exam, the proctor checked in each student with a $360^{\circ}$ view of the student's workspace and ID verification. Throughout the exam, students maintained audio and visual connections. Each proctor observed up to 24 students and logged any suspicious activities, which were reported to the Office of Academic Affairs. If needed, the College's coordinator of testing joined Zoom sessions to intervene on any suspicious activities in real-time using a breakout room to avoid interrupting other students. In addition, IT staff were available to help with any technical issues during the exam.

Computerized composite exams at UTHSC are administered every two weeks (eight total exams per semester) with questions from several courses combined into a single three-hour exam, and has been previously described. ${ }^{7}$ In both spring 2019 and spring 2020, all courses included on the composite exam had three to four questions per lecture hour. This study was considered exempt from review by the UTHSC Institutional Review Board.

The study population of interest included P1 $(n=192)$ and P2 $(n=195)$ students enrolled at UTHSC College of Pharmacy in spring 2020. Cohorts of P1 $(n=203)$ and P2 $(n=165)$ students enrolled at UTHSC College of Pharmacy in spring 2019 served as historical comparators. Remote proctoring began with exam 6 in the spring 2020 semester for both P1 and P2 students. To assess whether remote proctoring impacted academic performance, spring 2020 scores for exams 6,7 , and 8 were compared to that of a historical cohort proctored live in spring 2019. Academic performance on exams 1 through 4 was also compared between the two cohorts to evaluate year-to-year variation in performance during nonremote circumstances. Student scores on all composite exams administered in both semesters were collected retrospectively. Student demographics, including age, gender, and race were also obtained retrospectively.

Descriptive statistics for all variables were calculated. Shapiro Wilks tests and scatter plots were examined to assess normality of data. Mann Whitney tests compared the distribution of scores on each of the composite exams administered in spring 2020 to its corresponding exam administered in spring 2019. All tests were two-tailed, and $p$ values $<.05$ were considered statistically significant. All analysis was conducted with IBM SPSS Statistics for Windows, Version 25.0 (IBM Corporation, Armonk, NY).

\section{RESULTS}

Characteristics of the study participants in the remote proctoring and live proctoring cohorts are presented in Table 1. Most participants were female and white with a mean age of approximately 25 years old. There were significant differences in mean age for P2 students $(p<.001)$ and significant differences in race for P1 students $(p=.008)$ observed between the spring 2020 and spring 2019 cohorts.

All composite exam scores for spring 2020 and spring 2019 are presented in Table 2. Exams 1 through 4 were administered in 2019 and 2020 with live proctoring for both P1 and P2 students, and no significant differences in academic performance were noted on 4 of 8 (50\%) exams. As compared to spring 2019, spring 2020 academic performance was significantly worse on P1 exam 1 ( $p=.041), \mathrm{P} 1$ exam $2(p<.001)$, and P2 exam 1 ( $p<.001)$ but significantly better on $\mathrm{P} 2$ exam $2(p=.015)$. Median scores on exam 5 were significantly higher in spring 2020 versus spring 2019 for both P1 $(p=.016)$ and P2 ( $p<.001)$ students. Academic performance was significantly worse in spring 2020 versus spring 2019 for P1 exam 6 ( $p<.001$ ), but no significant difference was noted for P1 exam 7 or exam 8 . Median scores for P2 exam 6 and exam 8 were significantly higher in spring 2020 as compared to spring 2019 ( $p=.036$ and $p<.001$, respectively), but scores were significantly lower in spring 2020 for P2 exam 7 ( $p<.001)$.

\section{DISCUSSION}

This study aimed to examine the effects of remote proctoring on exam performance among P1 and P2 student pharmacists. The results indicate a variable impact on exam scores. On the first composite exam with remote proctoring, academic performance declined for P1 students but not P2 students. P1 students may have been more nervous about the proctoring modality change. Additionally, P2 students may be more accustomed to the remote learning technology since Zoom is used throughout the Doctor of Pharmacy (PharmD) program to facilitate communications between the three campuses even prior to the pandemic. After the first exam with remote proctoring, P1 student performance did not differ significantly from the historical cohort, suggesting there may be an adjustment period. 
For educators who are considering adopting a similar process for remote proctoring, we highly recommend including an orientation for both students and proctors and the opportunity to participate in a mock exam process before the first remotely proctored exam. Based on our experience in spring 2020, we added a practice remote proctoring session to acquaint students with the process beforehand for our students matriculating in fall 2020, and many upperclassmen commented that they wish this had been provided for them. In addition, we believe the human resources utilized in our approach are vital to ensure success. On exam days, four information technology staff and one academic affairs staff were available if any issues arose during the exam. In addition, a faculty member within academic affairs monitored the success of the remote proctoring process and followed up on any reports from the proctors of academic dishonesty.

When exams must be administered remotely, institutions often consider remote proctoring as an option to reduce academic dishonesty. Academic dishonesty was a concern voiced by several faculty and students at our institution during this transition to remote learning, especially after exam 5 was administered without any proctoring and significant improvements on exam scores were noticed. Substantive changes in exam design, such as using essays or other alternatives to multiple-choice questions, were not possible due to our large class size, limited resources, and a constrained timeline. Developing and implementing these remote proctoring procedures allowed our program to maintain our assessment approach in a secure manner. Furthermore, we were able to remotely proctor exams without any additional cost to the student because our procedures only utilized technology already in place prior to the pandemic and faculty and staff already employed by our institution.

Although remote proctoring offers benefits of reducing academic dishonesty, there are also potentially negative implications to consider. Remote proctoring may be considered intrusive by some students. During our experience, only a few students voiced these concerns, specifically regarding the check-in procedures; these students were offered the option to be checked in first so that their classmates did not see a $360^{\circ}$ view of their space. From an inclusivity perspective, remote proctoring requires a high-speed, stable internet connection and a device with video capabilities, and all students may not have access to these. Such students were offered the option to test on campus, and a video capable device was loaned to any students needing them. Based upon unsolicited feedback from students, the convenience benefits, including being able to choose where to take the exam, outweighed the negative parts of the experience, and students generally enjoyed the autonomy offered by remote proctoring.

This study is not without limitations. There may be confounding factors affecting exam performance that could not be elucidated by this study. One potential confounding factor includes the program's simultaneous change to remote instructional delivery in spring 2020, with some lectures delivered synchronously and others asynchronously, depending upon lecturer and course director. However, the course content, exam content, and exam construction (ie, multiple choice) did not change with the transition to remote learning in spring 2020 and were the same as that of spring 2019. Another limitation to consider is potential differences in the academic ability of the two cohorts. Academic performance on most exams administered in spring 2020 prior to the transition to remote learning (ie, exams 1 through 4) was observed to be comparable to that of the historical cohort, which lessens the potential impact of this limitation. The generalizability of the study may also be limited as the sample only includes students at one college of pharmacy. However, the likelihood of sample bias is low given that the characteristics of our study sample reflects the broader population of student pharmacists in the US.

Although this study was conducted amidst a global pandemic, the implications are relevant in a post-pandemic environment. Distance education and online learning programs are becoming more common. There are currently five PharmD programs in the US offering distance pathway options, which is an increase from just three programs in the previous year. ${ }^{8,9}$ Strategies to reduce academic dishonesty on high stakes exams taken remotely while also minimizing any negative impact on a student's performance will be paramount with distance pathway options increasing. Standard 10.17 (Academic Integrity) in the Accreditation Council for Pharmacy Education's 2016 Standards further emphasizes the importance of minimizing academic misconduct opportunities for all, including distance students. ${ }^{10}$ Based on the findings from our study, remote proctoring may offer a satisfactory solution to consider.

\section{CONCLUSION}

Student pharmacist composite exam scores did not consistently improve or decline with our implementation of remote proctoring in spring 2020. Remote proctoring may be a reasonable strategy to assure academic honesty and security of testing content in a remote educational environment with minimal impact on a student's exam performance.

\section{REFERENCES}

1. Azulay Chertok IR, Barnes ER, Gilleland D. Academic integrity in the online learning environment for health sciences students. Nurse Educ Today. 2014;34(10):1324-1329. 
2. Woldeab D, Brothen T. 21st Century assessment: Online proctoring, test anxiety, and student performance. Int $J$ E-Learn Dist Educ. 2019;34(1):1-10.

3. Stauffer R, Pitlick J, Challen L. Impact of an electronic-based assessment on student pharmacist performance in a required therapeutics course. Curr Pharm Teach Learn. 2020;12(3):287-290.

4. Karim MN, Kaminsky SE, Behrend TS. Cheating, reactions, and performance in remotely proctored testing: an exploratory experimental study. J Bus Psychol. 2014;29(4):555-572.

5. Milone AS, Cortese AM, Balestrieri RL, Pittenger AL. The impact of proctored online exams on the educational experience. Curr Pharm Teach Learn. 2017;9(1):108-114.

6. Weiner JA, Hurtz GM. A comparative study of online remote proctored versus onsite proctored high-stakes exams. J Appl Test Tech. 2017;18(1):13-20.

7. McDonough SL, Alford EL, Finks SW, Parker RB, Chisholm-Burns MA, Phelps SJ. Student pharmacists' perceptions of a composite examination in their first professional year. J Am Pharm Educ. 2016;80(1):Article 4.

8. Pharmacy College Application Service. Enrollment Options. Published 2020; https://www.pharmcas.org/schooldirectory/explore-and-compare/enrollment-options?enrollment_options=3. Accessed January 22, 2021.

9. American Association of Colleges of Pharmacy. Pharmacy School Admission Requirements: Table 1 (Pharm.D. Programs). Published 2019; https://www.aacp.org/sites/default/files/2020-02/psar-19-20-table-1v3.pdf. Accessed January 22, 2021.

10. Accreditation Council for Pharmacy Education. Accreditation Standards and Key Elements for the Professional Program in Pharmacy Leading to the Doctor of Pharmacy Degree ("Standards 2016"). Published February 2015. https://www.acpe-accredit.org/pdf/Standards2016FINAL.pdf. Accessed January 22, 2021. 
Table 1. Student Demographic Characteristics in the Spring 2020 and Spring 2019 Cohorts

\begin{tabular}{|c|c|c|c|c|c|c|}
\hline & $\begin{array}{c}\text { P1 Spring } 2020 \\
(\mathrm{n}=192)^{\mathrm{a}}\end{array}$ & $\begin{array}{c}\text { P1 Spring } 2019 \\
(n=203)^{a}\end{array}$ & $p$ value & $\begin{array}{c}\text { P2 Spring } 2020 \\
(\mathrm{n}=195)^{\mathrm{a}}\end{array}$ & $\begin{array}{c}\text { P2 Spring } 2019 \\
(\mathrm{n}=165)^{\mathrm{a}}\end{array}$ & $p$ value \\
\hline Age, mean (SD) & $24.6(4.09)$ & $24.8(2.93)$ & $\mathrm{NS}^{\mathrm{b}}$ & $24.8(2.93)$ & $26.1(3.04)$ & $<.001^{\mathrm{b}}$ \\
\hline Gender, n (\%) & & & $\mathrm{NS}^{\mathrm{c}}$ & & & $\mathrm{NS}^{\mathrm{c}}$ \\
\hline Female & $125(65.1 \%)$ & $116(57.1 \%)$ & & $116(59.5 \%)$ & $110(66.7 \%)$ & \\
\hline Male & $65(33.9 \%)$ & $78(38.4 \%)$ & & $78(40.0 \%)$ & $51(30.9 \%)$ & \\
\hline Race, n (\%) & & & $.008^{c}$ & & & $\mathrm{NS}^{\mathrm{c}}$ \\
\hline White & $119(62.0 \%)$ & $136(67.0 \%)$ & & $136(69.7 \%)$ & $104(63.0 \%)$ & \\
\hline Minority & $69(35.9 \%)$ & $53(26.1 \%)$ & & $53(27.2 \%)$ & $55(33.3 \%)$ & \\
\hline
\end{tabular}

${ }^{\mathrm{a} D e m o g r a p h i c ~ d a t a ~ n o t ~ a v a i l a b l e ~ f o r ~ a l l ~ s t u d e n t s, ~ t h u s ~ t o t a l ~ d o e s ~ n o t ~ e q u a l ~} 100 \%$

bIndependent samples t-test was used to determine significance, defined as $p<.05$

${ }^{c}$ Chi-square was used to determine significance, defined as $p<.05$

$\mathrm{P} 1=$ first-year student pharmacist; $\mathrm{P} 2=$ second-year student pharmacist

Table 2. Composite Exam Scores in Spring 2020 Versus Spring 2019

\begin{tabular}{|c|c|c|c|c|c|}
\hline & \multicolumn{2}{|r|}{ Spring 2020} & \multicolumn{2}{|r|}{ Spring 2019} & \multirow[b]{2}{*}{$p$ value $^{\mathrm{a}}$} \\
\hline & $\mathbf{n}$ & Performance, median (IQR) & $\mathbf{n}$ & Performance, median (IQR) & \\
\hline P1 Exam 1 & 191 & $79.7(14.87)$ & 203 & $83.3(15.38)$ & .041 \\
\hline P1 Exam 2 & 192 & $80.4(12.49)$ & 203 & $87.3(11.11)$ & $<.001$ \\
\hline P1 Exam 3 & 192 & $84.3(14.08)$ & 203 & $83.3(11.54)$ & NS \\
\hline P1 Exam 4 & 192 & $78.3(12.91)$ & 203 & $77.1(15.71)$ & NS \\
\hline P1 Exam $5^{b}$ & 192 & $84.0(13.89)$ & 203 & $80.3(16.66)$ & .016 \\
\hline P1 Exam 6c & 190 & $76.4(16.21)$ & 203 & $83.3(13.63)$ & $<.001$ \\
\hline $\mathrm{P} 1 \mathrm{Exam} 7^{\mathrm{c}}$ & 190 & $78.5(15.38)$ & 203 & $77.6(13.79)$ & NS \\
\hline P1 Exam $8^{c}$ & 190 & $80.0(16.36)$ & 203 & $80.0(13.34)$ & NS \\
\hline P2 Exam 1 & 195 & $80.0(14.28)$ & 165 & $85.9(12.50)$ & $<.001$ \\
\hline P2 Exam 2 & 195 & $79.7(15.62)$ & 165 & $77.8(15.28)$ & .015 \\
\hline P2 Exam 3 & 195 & $81.7(11.26)$ & 165 & $81.3(8.75)$ & NS \\
\hline P2 Exam 4 & 195 & $82.4(13.23)$ & 165 & $83.8(10.30)$ & NS \\
\hline P2 Exam $5^{\text {b }}$ & 195 & $89.1(10.94)$ & 165 & 80.6 (13.89) & $<.001$ \\
\hline $\mathrm{P} 2 \mathrm{Exam} 6^{\mathrm{c}}$ & 195 & $88.3(11.66)$ & 165 & $86.7(8.33)$ & .036 \\
\hline $\mathrm{P} 2 \operatorname{Exam} 7^{\mathrm{c}}$ & 195 & $83.8(10.30)$ & 165 & $86.8(10.30)$ & $<.001$ \\
\hline P2 Exam $8^{c}$ & 195 & $85.4(14.59)$ & 165 & $80.8(11.54)$ & $<.001$ \\
\hline
\end{tabular}

${ }^{\mathrm{a}}$ Mann Whitney was used to determine significance, defined as $\mathrm{p}<.05$

${ }^{\mathrm{b}}$ Administered remotely without proctoring

${ }^{\mathrm{c}}$ Administered with remote proctoring

$\mathrm{P} 1=$ first-year student pharmacist; $\mathrm{P} 2=$ second-year student pharmacist 